\title{
Research on Neural Network PID Quadratic Optimal Controller in Active Magnetic Levitation
}

\author{
Zhongqiao Zheng ${ }^{*}, 1,2$, Xiaojing Wang ${ }^{1}$, Yanhong Zhang ${ }^{2}$ and Jiangsheng Zhang ${ }^{2}$ \\ ${ }^{1}$ School of Mechatronic Engineering and Automation, Shanghai University, No.149, Yanchang Road, Shanghai, 200072, \\ China \\ ${ }^{2}$ School of Electronic Information \& Electric Engineering, Changzhou Institute of Technology, No.299, Tongjiangnan \\ Road, Changzhou City, Jiangsu Province, 213002, China
}

\begin{abstract}
In response to the uncertainty, nonlinearity and open-loop instability of active magnetic levitation control system, a neural network PID quadratic optimal controller has been designed using optimum control theory. By introducing supervised Hebb learning rule, constraint control for positioning errors and control increment weighting are realized by adjusting weighting coefficients, using weighed sum-squares of the control increment and the deviation between actual position and equilibrium position of the rotor in active magnetic levitation system as objective function. The simulation results show that neural network PID quadratic optimal controller can maintain the stable levitation of rotor by effectively improving static and dynamic performances of the system, so as to maintain the stable levitation of rotor in active magnetic levitation system which has stronger anti-jamming capacity and robustness.
\end{abstract}

Keywords: Active magnetic levitation, Adaptive PID controller, Quadratic optimal, Robustness, Single neuron.

\section{INTRODUCTION}

Active magnetic bearings (AMBs) maintain the stable levitation of rotor using electromagnetic force without any mechanical contact between stator and rotor, characterized by no wear, no lubrication, no oil pollution, no mechanical noise and adjustable stiffness and damping, controllable supporting force as in [1]. AMBs have been applied in many fields at home and abroad such as $\mathrm{CNC}$ machine, spaceflight, robotic and so on. Controller is the core of the active magnetic levitation system, whose performance affects the stability and dynamic characteristics of the system.

The conventional PID algorithm widely used has difficulty in parameter tuning, so it cannot meet the higher demands in stability and anti-jamming of control system as in [2] and [3]. So the design of controller and the selection of control law have been one of the research hotspots in active magnetic levitation system. A kind of adaptive fuzzy PID controller in active magnetic levitation system was researched in [4]. A kind of advanced PID controller in active magnetic levitation system was researched in [5]. Advanced RBF neural network PID controller in active magnetic levitation system was designed in [6] and a variable universe fuzzy PID controller in active magnetic levitation system was researched in [7].

\footnotetext{
*Address correspondence to this author at the School of Mechatronic Engineering and Automation, Shanghai University, No.149, Yanchang Road, Shanghai, 200072, China; Tel: 0086-21-66132245; Fax: 0086-21-66132245; E-mail: zhongqiaozheng@yeah.net
}

Combined with nonlinear mapping ability of neural network, adaptive learning ability and optimum control theory, introducing supervised Hebb learning rule, a neural network PID quadratic optimal controller is designed using weighed sum-squares of control increment and output error as objective function to adjust weighting coefficients based on the uncertainty, nonlinearity and open-loop instability of active magnetic levitation control system. So the difficulty of tuning parameters in normal PID controller is solved and the stability and anti-jamming capacity of active magnetic levitation control system are improved.

\section{STRUCTURE OF ACTIVE MAGNETIC BEARING}

An active magnetic levitation system consists of sensor, rotor, axial magnetic bearing, radial magnetic bearing, thrust plate, drive motor and auxiliary bearing as shown in Fig. (1).

An active magnetic levitation system has five degrees of freedom, in which two radial bearings respectively have two degrees of freedom at two orthogonal directions and the axial bearing has one degree of freedom in axial direction. Axial bearing is installed at thrust plate to maintain the equilibrium of rotor in axial direction and two radial bearings are installed at both ends of rotor to maintain the equilibrium of rotor in horizontal and vertical directions. Two auxiliary bearings play a role of protecting rotor by temporarily supporting rotor at both ends of rotor when AMBs don't work or get out of control and rotor drops to the auxiliary bearings, which protect the system from damage, so air gaps between auxiliary bearings and rotor should be less than between magnetic bearings and rotor. 


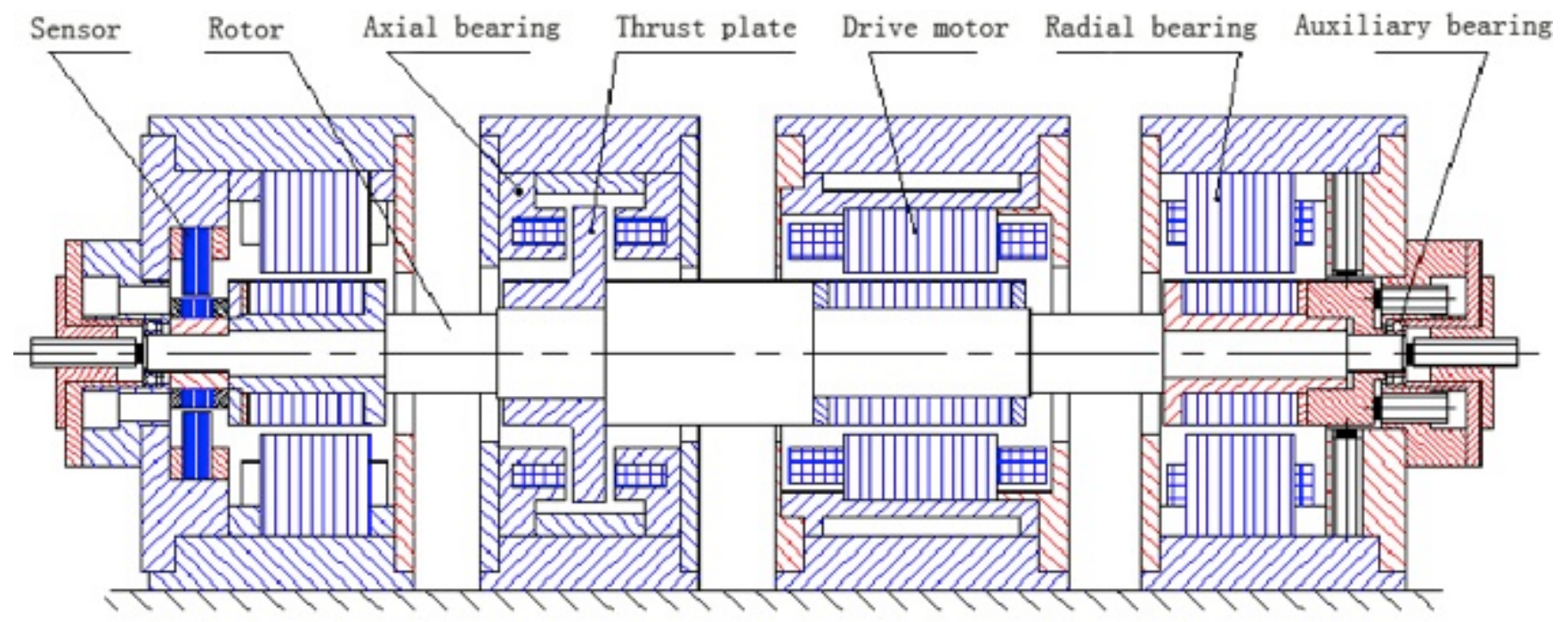

Fig. (1). Structure of active magnetic levitation system.

\section{MATHEMATICAL MODEL OF ACTIVE MAGNETIC LEVITATION SYSTEM}

For the convenience of research, the active magnetic levitation system with five degrees of freedom is simplified down to a system with one degree of freedom as research subject as shown in Fig. (2). The active magnetic levitation control system consists of displacement sensor, rotor, controller, power amplifier and electromagnet. The sensor should have higher conversion accuracy and stability as feedback element whose performance determines close-loop characteristic of the system, so eddy current sensor is adopted. The rotor is a controlled object whose equilibrium position and precision is controlled. As the core of active magnetic levitation system, the controller determines stiffness, damping of the system, the position and unbalance response of controlled object, whose designed objective is maintaining the stable non-contact equilibrium of controlled object. The power amplifiers transform control signal to control current of electromagnets to provide rotor with controllable attraction force.

In Fig. (2), $x_{0}$ is the equilibrium position of rotor, when offset of actual position is $x$, the displacement sensor can measure the change $x$ of the rotor position, and transform $x$ to electrical signal $U_{\mathrm{x}}$. $U_{\mathrm{x}}$ compares the expected voltage $U_{\mathrm{r}}$ corresponding to the stable levitation position of the rotor to obtain the error signal $U_{\mathrm{e}}$. The controller derives the control signal $U_{\mathrm{c}}$ from $U_{\mathrm{e}}$. The amplifier transforms the control signal to control current. In such a way, the current of the electromagnet is adjusted, the electromagnetic force is changed, and the stable levitation of the rotor is maintained.

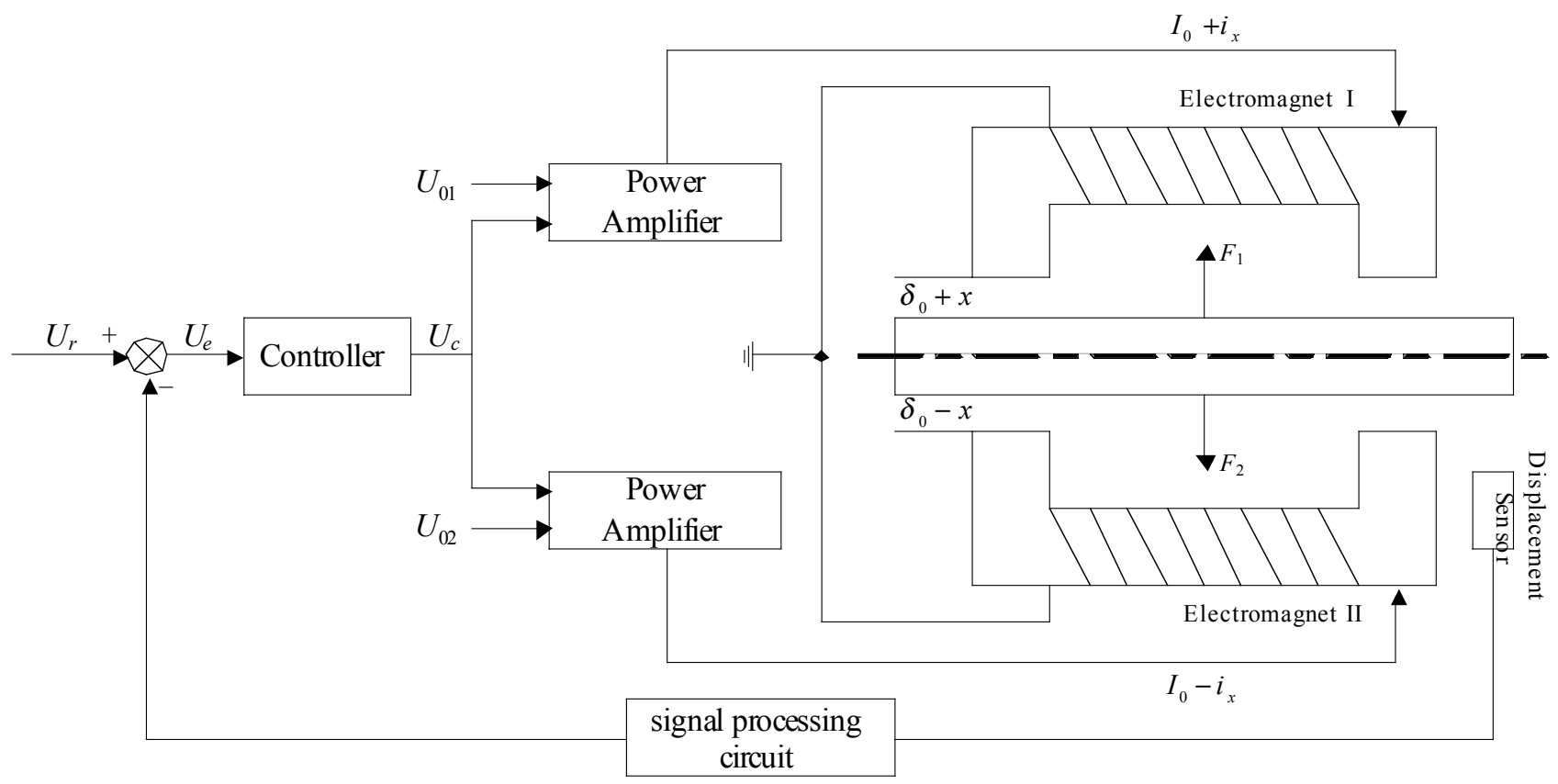

Fig. (2). Structure of single degree-of-freedom active magnetic levitation system. 
In Fig. (2), $U_{\mathrm{r}}$ is the input voltage value corresponding to equilibrium position of rotor. $U_{\mathrm{x}}$ is the voltage value corresponding to actual position of rotor, $U_{\mathrm{e}}=U_{\mathrm{r}}-U_{\mathrm{x}}$, which is an error signal. $U_{\mathrm{c}}$ is the output signal of adaptive neural network PID controller. $U_{0}$ is the voltage value which is bias currents of both upper and lower coils demand, $I_{0}$ is bias current component.

When neglecting leakage flux of the winding, reluctance of core and rotor, hysteresis loss and eddy-current loss of magnetic material occur. The resultant force of upper and lower electromagnets on the rotor will be zero when rotor whose mass is $m$, is on equilibrium position. When the rotor deviates from equilibrium position and the offset is $x$, to make the rotor to return back to equilibrium position, a control current $i$ must be added, which makes electromagnetic force of electromagnet I bigger and electromagnetic force of electromagnet II smaller. The electromagnetic force of both of electromagnets respectively is

$$
F_{1}=\frac{\mu_{0} s_{0} N^{2}\left(I_{0}+i\right)^{2}}{4\left(\delta_{0}+x\right)^{2}}, F_{2}=\frac{\mu_{0} s_{0} N^{2}\left(I_{0}-i\right)^{2}}{4\left(\delta_{0}-x\right)^{2}}
$$

where the meanings of the symbols are as follows:

$\mu_{0}$ - air permeability of ferromagnetic materials

$s_{0}$ - total pole face area

$N$ - number of turns per coil

$I_{0}$ - offset component of the current of the magnetic coil

$i$ - control current

$\delta_{0}$ - nominal air gap

$x$ - position displacement of the rotor center

The resultant force on the rotor is

$$
\Delta F_{x}=F_{1}-F_{2}=\frac{\mu_{0} s_{0} N^{2}}{4}\left[\left(\frac{I_{0}+i}{\delta_{0}+x}\right)^{2}-\left(\frac{I_{0}-i}{\delta_{0}-x}\right)^{2}\right]
$$

So

$m \ddot{x}=F_{1}-F_{2}$

Neglecting quadratic term and higher order term of $i$ and $x$, the Taylor series expansion of equation (3) can be obtained at the equilibrium point $x=0, i=0$ as follows:

$m \ddot{x}=\frac{\mu_{0} s_{0} N^{2} I_{0}^{2}}{\delta_{0}^{3}} x-\frac{\mu_{0} s_{0} N^{2} I_{0}}{\delta_{0}^{2}} i=C_{1} x-C_{2} i$ where

$C_{1}=\frac{\mu_{0} s_{0} N^{2} I_{0}^{2}}{\delta_{0}^{3}}$ - displacement stiff coefficient of active magnetic bearing system

$C_{2}=\frac{\mu_{0} s_{0} N^{2} I_{0}}{\delta_{0}^{2}}-$ voltaic stiff coefficient of active magnetic bearing system

Using Laplace transformation for equation (4), the following equation can be obtained

$m s^{2} X(s)=C_{1} X(s)-C_{2} I(s)$

The open-loop transfer function of the system can be obtained after rearranging

$G(s)=\frac{X(s)}{I(s)}=\frac{-C_{2}}{m s^{2}-C_{1}}$

The transfer function has a pole in the open right-half plane as shown from equation (6), which makes the openloop control system unstable.

\section{SELF-ADAPTIVE SINGLE NEURON PID QUADRATIC OPTIMAL CONTROLLER}

Incremental PID control algorithm can be expressed in difference equation as follows:

$$
\begin{aligned}
\Delta u(k)= & K_{p}[e(k)-e(k-1)]+K_{i} e(k) \\
& +K_{d}[e(k)-2 e(k-1)+e(k-2)]
\end{aligned}
$$

where $K_{\mathrm{p}}$ is proportion coefficient, $K_{\mathrm{i}}$ is integration coefficient, $K_{\mathrm{d}}$ is differentiation coefficient, $e(k)=r(k)-y(k)$, is the deviation value at $k$ sampling instant, $r(k)$ is expected value at $k$ sampling instant, $y(k)$ is the actual position value of rotor at $k$ sampling instant.

Block diagram of active magnetic levitation system based on self-adaptive single neuron PID controller is shown in Fig. (3).

In Fig. (3), $R$ is equilibrium position of rotor, $Y$ is actual position of rotor, $e$ is displacement deviation signal, $u$ is output signal of self-adaptive single neuron PID controller which is a voltage signal over coils of electromagnet, $x_{1}, x_{2}$, $x_{3}$ are input signals of network layer, $\Delta u$ is output signal of network layer. Adopting incremental PID control algorithm, self-adaptive single neuron PID controller realizes self-

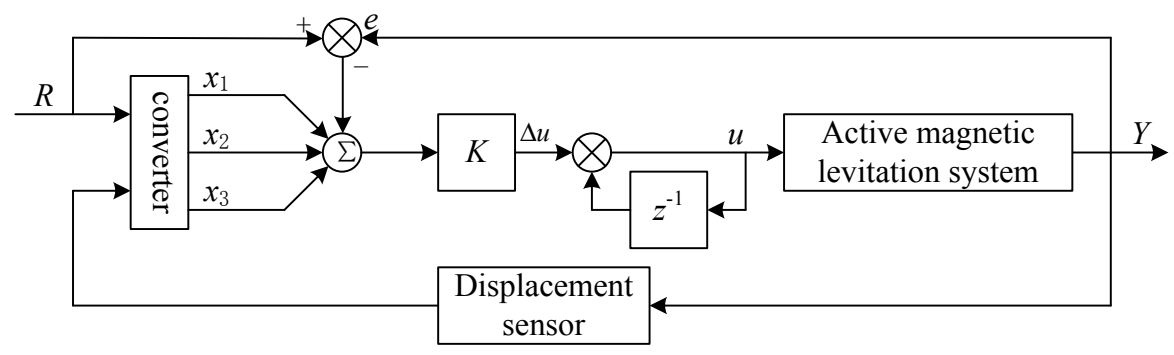

Fig. (3). Active magnetic levitation system based on Self-adaptive single neuron PID controller. 
adaptive and self-organizing functions by adjusting weighting coefficients. According to supervised Hebb learning rule and optimal control theory, adjusting weighting coefficients minimize weighed sum-squares of output error and control increment, to indirectly achieve constraint control for output error and control increment weighting by introducing quadratic performance index.

Quadratic performance index is shown as follows:

$J(k)=\frac{1}{2}[P(r(k)-y(k))]^{2}+Q \Delta^{2} u(k)$

where $r(k)$ and $y(k)$ are reference input signal and output signal at $k$ sampling instant, $u(k)$ is control signal, $P$ and $Q$, respectively is weighting coefficient of error signal and control increment.

The output of neuron is shown as follows:

$u(k)=u(k-1)+K \sum_{i=1}^{3} \bar{\omega}_{i}(k) x_{i}(k)$

where

$$
\begin{aligned}
& \bar{\omega}_{i}(k)=\frac{\omega_{i}(k)}{\sum_{i=1}^{3}\left|\omega_{i}(k)\right|} \\
& \omega_{1}(k)=\omega_{1}(k-1)+\eta_{I} K\left[a e(k) x_{1}(k)-K \sum_{i=1}^{3}\left(\omega_{i}(k) x_{i}(k)\right] x_{1}(k)\right. \\
& \omega_{2}(k)=\omega_{2}(k-1)+\eta_{P} K\left[a e(k) x_{2}(k)-K \sum_{i=1}^{3}\left(\omega_{i}(k) x_{i}(k)\right] x_{2}(k)\right. \\
& \omega_{3}(k)=\omega_{3}(k-1)+\eta_{D} K\left[a e(k) x_{3}(k)-K \sum_{i=1}^{3}\left(\omega_{i}(k) x_{i}(k)\right] x_{3}(k)\right. \\
& x_{1}(k)=e(k) \\
& x_{2}(k)=e(k)-e(k-1) \\
& x_{3}(k)=e(k)-2 e(k-1)+e(k-2)
\end{aligned}
$$

$\eta_{\mathrm{P}}, \eta_{\mathrm{I}}, \eta_{\mathrm{D}}$ respectively are learning rate of proportion, integral and differential term, $\mathrm{K}$ is proportion coefficient of neuron. Proportion, integral and differential term of PID controller respectively use different learning rates, which can realize to respectively adjust different weighting coefficients.

The following equation can be obtained by comparing equations (7) and (8)

$$
\begin{aligned}
& K_{p}=K \frac{\omega_{2}(k)}{\sum_{i=1}^{3} \omega_{i}(k) x_{i}(k)}= \\
& K \frac{\omega_{2}(k-1)+\eta_{P} K\left[a e(k) x_{2}(k)-K \sum_{i=1}^{3}\left(\omega_{i}(k) x_{i}(k)\right] x_{2}(k)\right.}{\sum_{i=1}^{3} \omega_{i}(k) x_{i}(k)}
\end{aligned}
$$

$$
\begin{aligned}
& K_{i}=K \frac{\omega_{1}(k)}{\sum_{i=1}^{3} \omega_{i}(k) x_{i}(k)}= \\
& K \frac{\omega_{1}(k-1)+\eta_{I} K\left[a e(k) x_{1}(k)-K \sum_{i=1}^{3}\left(\omega_{i}(k) x_{i}(k)\right] x_{1}(k)\right.}{\sum_{i=1}^{3} \omega_{i}(k) x_{i}(k)} \\
& K_{d}=K \frac{\omega_{3}(k)}{\sum_{i=1}^{3} \omega_{i}(k) x_{i}(k)}= \\
& K \frac{\omega_{3}(k-1)+\eta_{D} K\left[a e(k) x_{3}(k)-K \sum_{i=1}^{3}\left(\omega_{i}(k) x_{i}(k)\right] x_{3}(k)\right.}{\sum_{i=1}^{3} \omega_{i}(k) x_{i}(k)}
\end{aligned}
$$

When active magnetic levitation system is affected by the disturbance, the rotor deviates from equilibrium position. When downward offset is $x$, the distance between upper coils of electromagnet and rotor increases, displacement sensor can be actual position of rotor and transform it to corresponding voltage signal. Error signal can be obtained by comparing above signal with the voltage signal corresponding to equilibrium position of rotor, and enter neuron network that tunes $K_{\mathrm{p}}, K_{\mathrm{i}}$ and $K_{\mathrm{d}}$ of PID controller to achieve the optimization and generates control signal by correlation and searching. In this way, the current of upper coils increases and of lower coils decreases, which makes electromagnetic force F1 of upper coils increase, and slowly move rotor upward to equilibrium position.

\section{SYSTEM SIMULATION AND ANALYSIS}

In active magnetic levitation system of this paper, the mass $m$ of rotor is $10 \mathrm{~kg}$, the equilibrium position $x_{0}$ is $0.3 \times 10^{-3} \mathrm{~m}$, offset component $I_{0}$ of the current of the magnetic coil is $2.0 \mathrm{~A}$, air permeability $\mu_{0}$ is $4 \pi \times 10^{-7} \mathrm{Vs} / \mathrm{Am}$, total pole face area $\mathrm{s}_{0}$ is $340 \mathrm{~mm}^{2}$, number of turns per coil $N$ is 190. Plugging these parameters into equation (6), openloop transfer function of active magnetic levitation control system can be obtained as follows:

$$
G(s)=-\frac{34}{s^{2}-22.85 \times 10^{4}}
$$

It is established that the input signal of active magnetic levitation system is a unit step signal, the learning rate of proportion, integral and differential term respectively is $\eta_{\mathrm{P}}=$ $100, \eta_{\mathrm{I}}=3, \eta_{\mathrm{D}}=150$, proportion coefficient of neuron $K$ is 0.02 , error weighting coefficient $P$ is 1 , control increment weighting coefficient $Q$ is 1 in performance index function. At $\mathrm{t}=0.2 \mathrm{~s}$, the system is added into a disturbance signal, whose magnitude is 0.05 , tuning curves of $K_{\mathrm{p}}, K_{\mathrm{i}}$ and $K_{\mathrm{d}}$ are shown in Fig. (4), output of the system is shown in Fig. (5), and curve of performance index is shown in Fig. (6) by simulation using MATLAB as in [8-10]. 
From Fig. (4), it can be observed that three parameters of self-adaptive single neuron PID quadratic optimal controller respectively is $K_{\mathrm{p}}=0.3026, K_{\mathrm{i}}=0.3171, K_{\mathrm{d}}=0.2740 . K_{\mathrm{p}}$ increases greatly in the initial stages to accelerate system response, at which time $K_{\mathrm{i}}$ increases obviously to improve static performance of the system, and the system slowly becomes stable. Correspondingly $K_{\mathrm{d}}$ also increases obviously to improve dynamic performance of the system and shorten the adjusting time, $K_{\mathrm{p}}, K_{\mathrm{i}}$ and $K_{\mathrm{d}}$ tend to become stable when the value of $\mathrm{t}=0.02 \mathrm{~s}$.

From Fig. (5), it can be observed that overshoot of the system is very small, after $t=0.05 \mathrm{~s}$ so that output of the system can accurately track input signal. In other words, rotor can accurately return to equilibrium position, and after $0.01 \mathrm{~s}$ external disturbance can be eliminated to make rotor return to equilibrium position. The control system has stronger anti-jamming capacity and better static and dynamic characteristics.

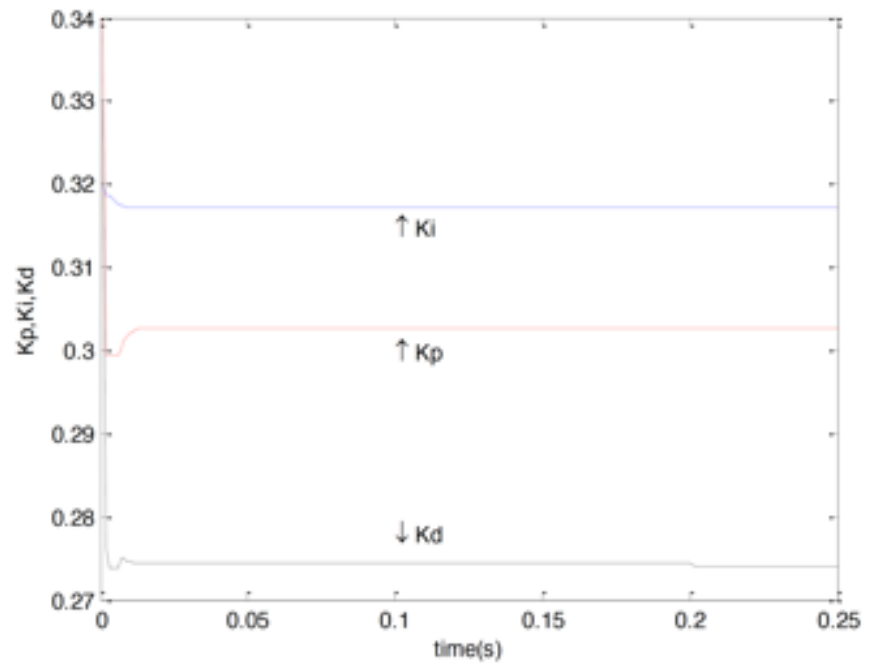

Fig. (4). Output value of parameter.

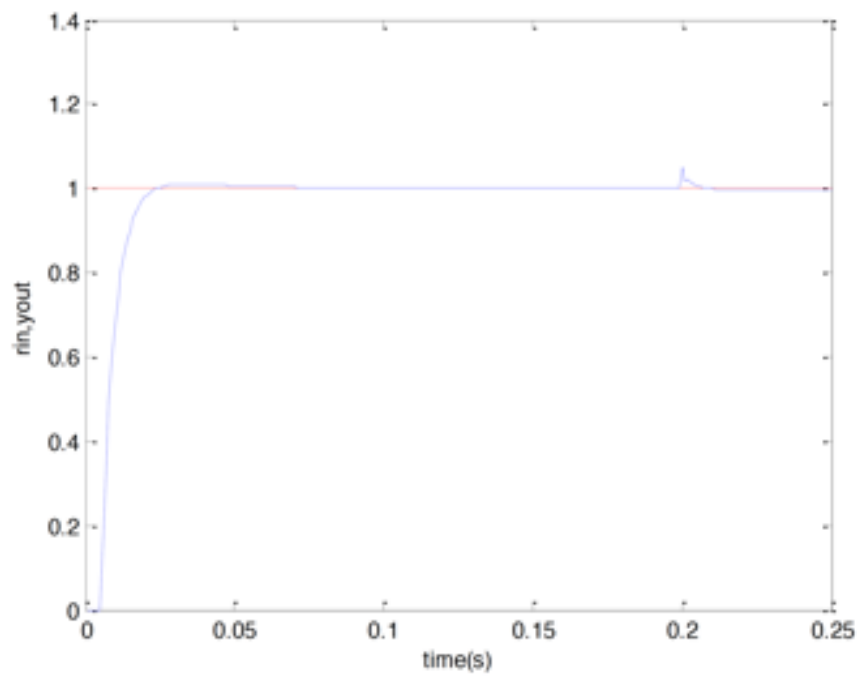

Fig. (5). Output of the system.

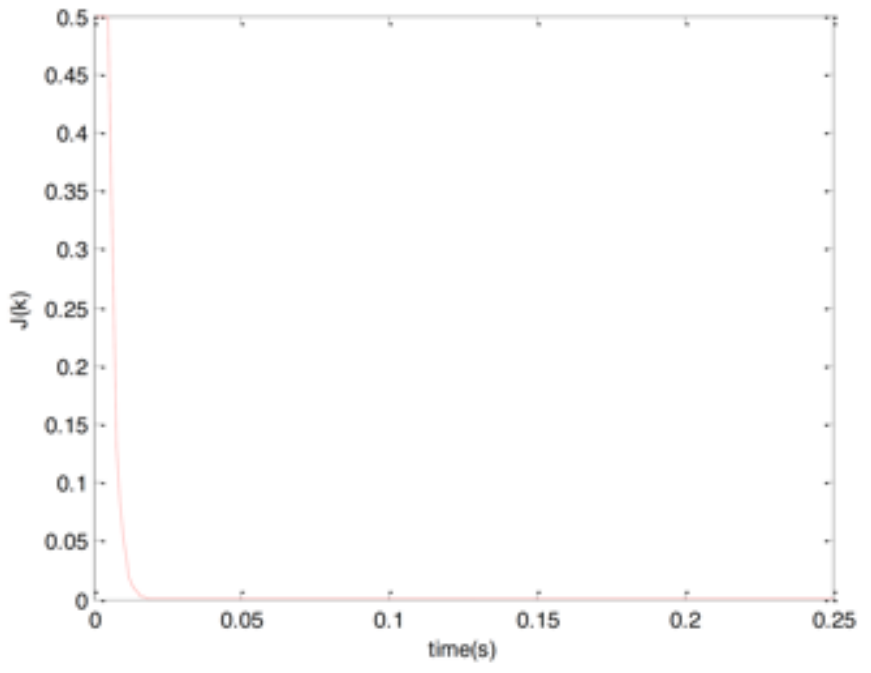

Fig. (6). Curves of performance index $J(k)$.

From Fig. (6), it can be observed that performance index $J(k)$ of optimization is close to zero after $\mathrm{t}=0.02 \mathrm{~s}$, at which time active magnetic levitation system reaches static state.

\section{CONCLUSION}

According to the uncertainty, nonlinearity and open-loop instability of active magnetic levitation control system, combining identification theory of neural network, optimum control theory and conventional PID control, a single neuron PID quadratic optimal controller is designed. Based on selfadaptive single neuron PID controller, quadratic optimal performance index is introduced to realize to tune parameters on line by minimizing weighed sum-squares of control increment and position error of rotor to adjust weighting coefficients. The simulation results show that the active magnetic levitation system based on self-adaptive single neuron PID quadratic optimal controller has better stability and robustness.

\section{CONFLICT OF INTEREST}

The authors confirm that this article content has no conflict of interest.

\section{ACKNOWLEDGEMENTS}

We acknowledge the help of Bing Zhang and Wensheng Huang in designing the controller. We also thank the anonymous reviewers for their numerous helpful suggestions. The project was supported by the National Nature Science Foundation of China (No. 51175052) and Applied Basic Research Programs of Changzhou City (No. CJ20130014), also supported by Scientific Research Fund of Changzhou Institute of Technology (No. YN1216), respectively.

\section{REFERENCES}

W.K.S. Khoo, K. Kalita, S.D. Garvey, R. J. Hill-Cottingham, D. Rodger, and J.F. Eastham, "Active Axial-Magnetomotive Force Parallel-Airgap Serial Flux Magnetic Bearings," IEEE Transactions on Magnetics, vol. 46, no. 7, pp. 2596-2602, 2010. 
[2] J. Ritonja, B. Polajzer, D. Dolinar, B. Grcar, and P. Cafuta, "Active Magnetic Bearings Control", In: Proceedings of the 29th Chinese Control Conference, 2010, pp. 5604-5609.

[3] T. Schuhmann, W. Hofmann, and R. Werner, "Improving operational performance of active magnetic bearings using kalman filter and state feedback control," IEEE transactions on industrial electronics, vol. 59, no. 2, 2012.

[4] Z. Jing, and X. Lin, "Fuzzy adaptive PID control in magnetic levitation system," Journal of Computer Applications, vol. 29, pp. 329-334, 2009.

[5] S. Kai, and S. Chunxiang, "Research on an active magnetic suspension system with Matlab simulation," Industrial Instrumentation \& Automation, issues 4, pp. 7-14, 2008.

[6] Z. Jing, P. Xuehong, and X. Haifeng, "Improved RBF Neural
Network Control of Magnetic Levitation," Journal of Harbin University of Science and Technology, vol.16, pp. 48-52, 2011.

[7] W. Xiaoling, and Z. Guangming, "Magnetic levitating bearing control system simulation based on variable universe fuzzy PID algorithm," Instrument Technique and Sensor, issues 12, pp. 144$147,2012$.

[8] L. Wenguang, H. Junfeng, and L. Hongli, "Efficient simulation of fuzzy control system based on MATLAB (SIMULINK)," Computer Simulation, vol.18(3), pp. 15-16, 2001.

[9] E. Shameli, M.B. Khamesee, and J.P. Hu Issoon, "Nonlinear controller design for a magnetic levitation device", Microsystem Technologies, vol. 13, pp. 831- 835, 2003.

[10] L. Jinkun, "Advanced PID Control and MATLAB Simulation", 1st ed., Beijing: Publishing House of Electronics Industry, 2003.

(c) Zheng et al.; Licensee Bentham Open.

This is an open access article licensed under the terms of the Creative Commons Attribution Non-Commercial License (http://creativecommons.org/licenses/ by-nc/4.0/) which permits unrestricted, non-commercial use, distribution and reproduction in any medium, provided the work is properly cited. 